\title{
IN-SPACE CALIBRATION OF A GYRO QUADRUPLET
}

\author{
Itzhack Y. Bar-Itzhack ${ }^{1}$ and Richard R. Harman ${ }^{2}$ \\ Flight Dynamics Analysis Branch, Code 572 \\ Guidance Navigation and Control Center \\ NASA-Goddard Space Flight Center \\ Greenbelt, MD 20771
}

\begin{abstract}
This work presents a new approach to gyro calibration where, in addition to being used for computing attitude that is needed in the calibration process, the gyro outputs are also used as measurements in a Kalman filter. This work also presents an algorithm for calibrating a quadruplet rather than the customary triad gyro set. In particular, a new misalignment error model is derived for this case. The new calibration algorithm is applied to the EOS-AQUA satellite gyros. The effectiveness of the new algorithm is demonstrated through simulations.
\end{abstract}

\section{INTRODUCTION}

Gyro calibration as well as calibration of other instruments includes two stages. In the first stage the instrument error parameters are estimated. During the second stage those errors are continuously removed from the gyro readings. In the classical approach to gyro calibration, the gyro outputs are used to maintain or compute body orientation rather than being used as measurements in the context of filtering. In inertial navigation, for example (ref. 1), gyro errors cause erroneous computation of velocity and position, and then when the latter are compared to measured velocity and position, a great portion of the computed velocity and position errors can be determined. The latter errors are then fed into a Kalman filter (KF) that uses the Inertial Navigation System (INS) error model to infer on the gyro errors. Similarly, when applying the classical approach to spacecraft (SC) attitude determination, the gyro outputs are used to compute the attitude and then attitude measurements (refs. 2,3) are used to determine the attitude errors, which again using a KF, indicates what the gyro errors are.

In the approach adopted in this work, the gyro outputs are used as angular rate measurements and are compared to estimated angular rate measurements. However, this approach requires the knowledge of the angular rate. In the past (ref. 4), the estimated angular rate was computed in a rather simplistic way assuming basically that the rate was constant. In the present work, the estimated angular rate is derived using a KF whose input can be any kind of attitude measurement; therefore, the angular rate experienced by the SC can be continuously changing, and yet a good estimate of the rate, necessary for calibration, can be obtained.

The calibration algorithm presented in this work was derived for a set of quadruplet gyros. This required the derivation of a new error model, particularly for the gyro misalignments. The new calibration algorithm was applied to the gyro package of the EOS-AQUA satellite. The latter consists of four gyros, which are given the task of measuring the three components of the SC angular velocity vector resolved in the body Cartesian coordinates.

In the next section the gyro error model is derived. The section that follows presents an algorithm for computing the calibration parameters when the rate is known, and then in the section that follows we

\footnotetext{
${ }^{1}$ Sophie and William Shamban Professor of Aerospace Engineering. On sabbatical leave from the Faculty of Aerospace Engineering, Technion-Israel Institute of Technology. Member Technion Asher Space Research Institute. NRC NASA Resident Research Associate. Tel: (301) 286-9216, Fax: (301) 286-036, Email: ibaritz@pop500.gsfc.nasa.gov

${ }^{2}$ Aerospace Engineer. Tel: (301) 286-5125, Fax: (301) 286-036,

Email: richard.r.harman.1@gsfc.nasa.gov
} 
present the same when the rates are unknown. In the following section we present the compensation procedure that needs to take place to complete the calibration process, and in the subsequent section we present simulation results. Finally, in the last section, the conclusions are presented.

\section{GYRO ERROR MODEL}

The gyro errors that are considered in this work are: misalignment, scale factor error, and bias (constant drift rate). The gyro error model is basically a linear model, which associates small error sources to the gyro outputs. Due to the linearity of the model we can compute the contribution of each error source independently, and then sum up all the contributions into one linear model.

We start the description of the error model, by deriving the expression for the gyro misalignments.

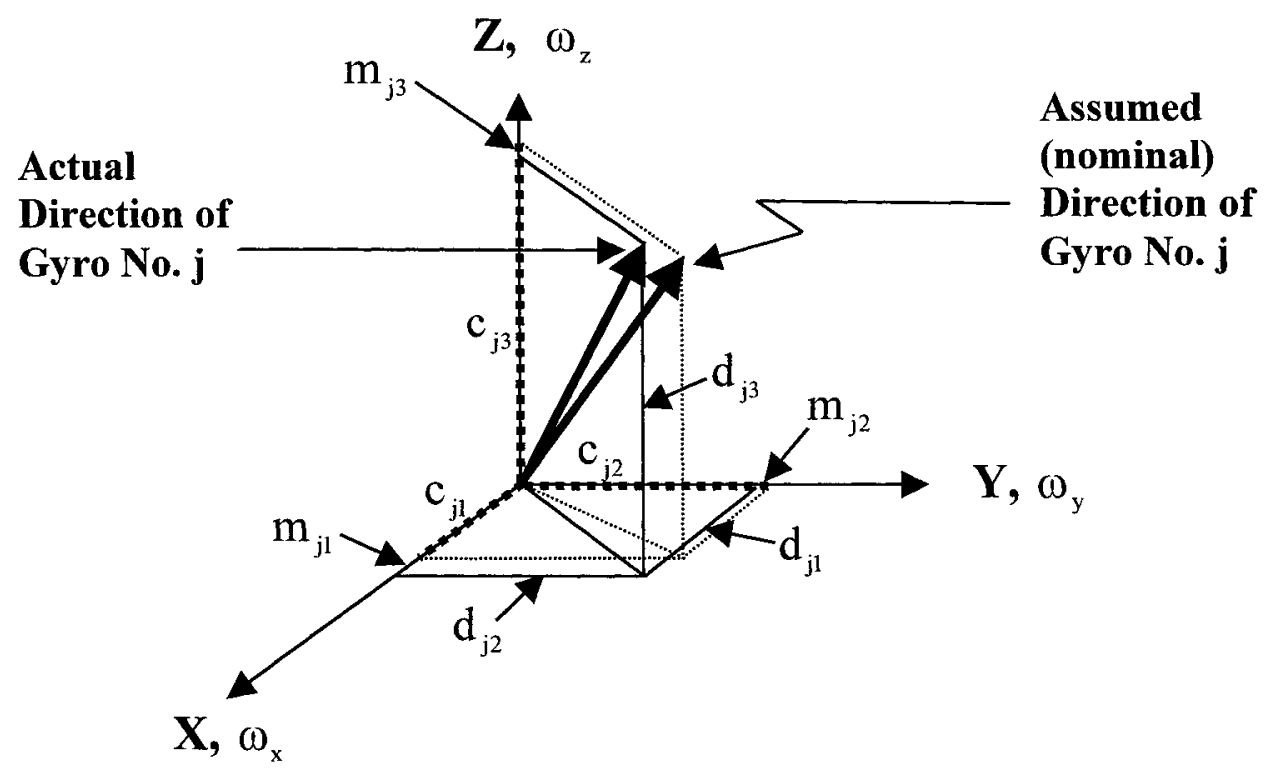

Fig. 1: The geometry of the Assumed and the Actual Direction of the Gyro Input Axis

\section{Misalignment Model}

The assumed direction of the sensitive axis of gyro $\mathrm{j}$, which is one of the four gyros, is presented in Fig. 1 where the body coordinate axes are also presented and are denoted by $\mathbf{X}, \mathbf{Y}$, and $\mathbf{Z}$. The orientation of this gyro is expressed by a vector of unit length in the direction of the gyro sensitive axis. The direction of this unit vector in the body coordinates is expressed by its three direction cosines, which are identical to its components when the unit vector is resolved in the body coordinates. These components are $\mathrm{c}_{\mathrm{j} 1}, \mathrm{c}_{\mathrm{j} 2}$, and $\mathrm{c}_{\mathrm{j} 3}$. Being direction cosines, or equivalently, components of a unit vector, the sum of their squares adds up to 1 ; that is,

$$
c_{j 1}^{2}+c_{j 2}^{2}+c_{j 3}^{2}=1
$$

The rate that this gyro reads is the projection of the angular velocity vector on this unit vector. If we express the angular velocity vector in the body coordinates, where its components are $\omega_{\mathrm{x}}, \omega_{\mathrm{y}}$, and $\omega_{\mathrm{z}}$, then this projection is given by

$$
\mathbf{1}_{\mathrm{j}} \cdot \boldsymbol{\omega}=\mathrm{c}_{\mathrm{j} 1} \omega_{\mathrm{x}}+\mathrm{c}_{\mathrm{j} 2} \omega_{\mathrm{y}}+\mathrm{c}_{\mathrm{j} 3} \omega_{\mathrm{z}}
$$


where $\mathbf{1}_{j}$ is the unit vector along the $j^{\text {th }}$ gyro sensitive axis, and $\boldsymbol{\omega}$ is the angular rate vector. The nominal (error-less) reading of this gyro is then

$$
G_{j n}=c_{j 1} \omega_{x}+c_{j 2} \omega_{y}+c_{j 3} \omega_{z}=\left[\begin{array}{lll}
c_{j 1} & c_{j 2} & c_{j 3}
\end{array}\right]\left[\begin{array}{c}
\omega_{x} \\
\omega_{y} \\
\omega_{z}
\end{array}\right]
$$

where the subscript $n$ denotes the nominal or design value. Combining all four gyros we obtain

$$
\left[\begin{array}{l}
G_{1 n} \\
G_{2 n} \\
G_{3 n} \\
G_{4 n}
\end{array}\right]=\left[\begin{array}{lll}
c_{11} & c_{12} & c_{13} \\
c_{21} & c_{22} & c_{23} \\
c_{31} & c_{32} & c_{33} \\
c_{41} & c_{42} & c_{43}
\end{array}\right]\left[\begin{array}{l}
\omega_{x} \\
\omega_{y} \\
\omega_{z}
\end{array}\right]
$$

Define

$$
\mathbf{G}_{n}^{T}=\left[\begin{array}{llll}
G_{1 n} & G_{2 n} & G_{3 n} & G_{4 n}
\end{array}\right]
$$

and

Eq. (3.a) can be written as

$$
\mathrm{C}=\left[\begin{array}{lll}
\mathrm{c}_{11} & \mathrm{c}_{12} & \mathrm{c}_{13} \\
\mathrm{c}_{21} & \mathrm{c}_{22} & \mathrm{c}_{23} \\
\mathrm{c}_{31} & \mathrm{c}_{32} & \mathrm{c}_{33} \\
\mathrm{c}_{41} & \mathrm{c}_{42} & \mathrm{c}_{43}
\end{array}\right]
$$

$$
\mathbf{G}_{\mathrm{n}}=\mathrm{C} \boldsymbol{\omega}
$$

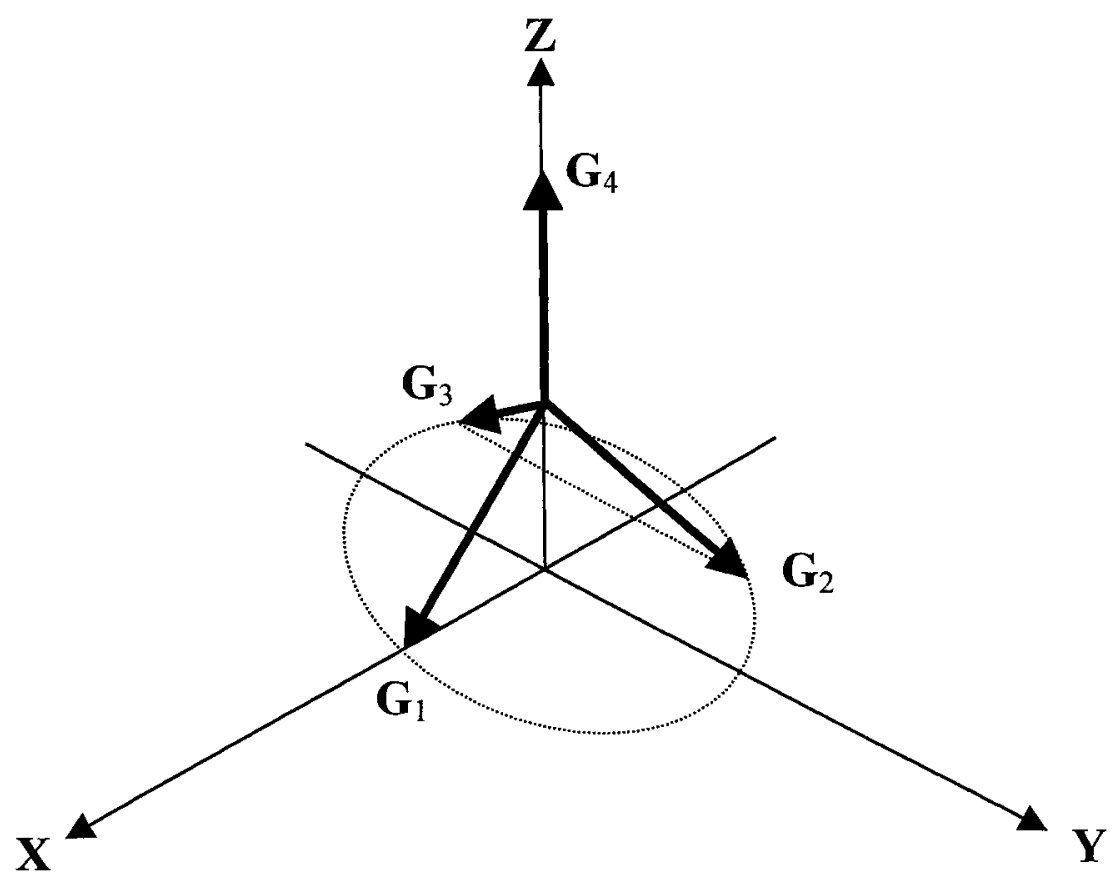

Fig. 2: The Gyro Configuration in the EOS-AQUA Satellite. 
In the EOS-AQUA satellite the gyro configuration is as shown in Fig. 2 where, as mentioned before, $\mathbf{X}, \mathbf{Y}$, and $\mathbf{Z}$ are the axes of the body frame. The $\mathrm{C}$ matrix in this case is

$$
C=\left[\begin{array}{ccc}
\sqrt{\frac{2}{3}} & 0 & -\sqrt{\frac{1}{3}} \\
-\sqrt{\frac{1}{6}} & \sqrt{\frac{1}{2}} & -\sqrt{\frac{1}{3}} \\
-\sqrt{\frac{1}{6}} & -\sqrt{\frac{1}{2}} & -\sqrt{\frac{1}{3}} \\
0 & 0 & 1
\end{array}\right]
$$

Note that the vector described by each row is of unit length like it should be (see Eq. 1).

Due to misalignment, the sensitive axis of each gyro may actually point at a slightly different direction than the assumed one. This is illustrated in Fig. 1 where the components of this direction (which is still a unit vector) are $d_{j 1}, d_{j 2}$, and $d_{j 3}$ respectively. Following the steps that led to the development of the nominal gyro reading presented in Eq. (2.b), the actual gyro reading is found to be

$$
G_{j a}=d_{j 1} \omega_{x}+d_{j 2} \omega_{y}+d_{j 3} \omega_{z}=\left[\begin{array}{lll}
d_{j 1} & d_{j 2} & d_{j 3}
\end{array}\right]\left[\begin{array}{c}
\omega_{x} \\
\omega_{y} \\
\omega_{z}
\end{array}\right]
$$

where the subscript $a$ denotes an actual value. The difference, $\Delta \mathrm{G}_{\mathrm{j}}$, between the reading of the $\mathrm{j}$ th gyro and its assumed nominal reading is computable using Eqs. (2.b) and (6), as follows;

$$
\Delta G_{j}^{m}=G_{j a}-G_{j n}=\left[\begin{array}{lll}
d_{j 1}-c_{j 1} & d_{j 2}-c_{j 2} & d_{j 3}-c_{j 3}
\end{array}\right]\left[\begin{array}{l}
\omega_{x} \\
\omega_{y} \\
\omega_{z}
\end{array}\right]
$$

where the superscript $m$ denotes the fact that the error is due to misalignment. We denote by $\mathrm{m}_{\mathrm{ji}}$ the differences $d_{j 1}-c_{j 1}, d_{j 2}-c_{j 2}$, and $d_{j 3}-c_{j 3}$ as follows

$$
\mathrm{m}_{\mathrm{j} 1}=\mathrm{d}_{\mathrm{j} 1}-\mathrm{c}_{\mathrm{j} 1} \text { (8.a) } \quad \mathrm{m}_{\mathrm{j} 2}=\mathrm{d}_{\mathrm{j} 2}-\mathrm{c}_{\mathrm{j} 2} \quad \text { (8.b) } \quad \mathrm{m}_{\mathrm{j} 3}=\mathrm{d}_{\mathrm{j} 3}-\mathrm{c}_{\mathrm{j} 3}
$$

Using Eqs. (8) we can write Eq. (7), as follows;

$$
\Delta \mathrm{G}_{\mathrm{j}}^{\mathrm{m}}=\left[\begin{array}{lll}
\omega_{\mathrm{x}} & \omega_{\mathrm{y}} & \omega_{\mathrm{z}}
\end{array}\right]\left[\begin{array}{l}
\mathrm{m}_{\mathrm{j} 1} \\
\mathrm{~m}_{\mathrm{j} 2} \\
\mathrm{~m}_{\mathrm{j} 3}
\end{array}\right]
$$

The $\mathrm{m}_{\mathrm{ji}}$ differences are shown in Fig. 1. Actually only two of the $\mathrm{m}_{\mathrm{ji}}$ of each gyro are independent. This results from the fact that the nominal as well as the actual directions of the gyros are given by vectors of unit length. For a reason that will be clear later, let us choose to present the third component of $\mathrm{m}_{\mathrm{j}}$ by the 
first two; that is, we express $\mathrm{m}_{\mathrm{j} 3}$, the misalignment along the sensitive axis, by $\mathrm{m}_{\mathrm{j} 1}$ and $\mathrm{m}_{\mathrm{j} 2}$. Since similarly to Eq. (1), it is also true that

$$
d_{j 1}^{2}+d_{j 2}^{2}+d_{j 3}^{2}=1
$$

then using this relation and Eq. (8.c) we can write

$$
\mathrm{m}_{\mathrm{j} 3}=\sqrt{1-\mathrm{d}_{\mathrm{j} 1}^{2}-\mathrm{d}_{\mathrm{j} 2}^{2}}-\sqrt{1-\mathrm{c}_{\mathrm{j} 1}^{2}-\mathrm{c}_{\mathrm{j} 2}^{2}}
$$

For the case described by the fourth gyro $\left(\mathrm{G}_{4}\right.$ in fig. 3$)$ the nominal direction of the gyro sensitive axis is along the $\mathrm{Z}$ axis; therefore, $\mathrm{c}_{41}=\mathrm{c}_{42}=0$, then from Eqs. (8.a) and (8.b)

and from Eq. (11)

$$
\mathrm{m}_{41}=\mathrm{d}_{41} \quad \text { (12.a) } \quad \mathrm{m}_{42}=\mathrm{d}_{42}
$$

$$
\mathrm{m}_{43}=\sqrt{1-\mathrm{d}_{41}^{2}-\mathrm{d}_{42}^{2}}-1
$$

In the case where the misalignments are small, $\mathrm{d}_{41}^{2}$ and $\mathrm{d}_{42}^{2}$ are small too. Therefore we can expand the square root function of Eq. (11) in a Taylor series, as follows;

$$
\sqrt{1-\mathrm{d}_{41}^{2}-\mathrm{d}_{42}^{2}}=1-\frac{1}{2} \mathrm{~d}_{41}^{2}-\frac{1}{2} \mathrm{~d}_{42}^{2}
$$

(Note that the linear term of the series vanishes). Substituting of the last equation into Eq. (13) yields

$$
\mathrm{m}_{43}=-\frac{1}{2} \mathrm{~d}_{41}^{2}-\frac{1}{2} \mathrm{~d}_{42}^{2}=-\frac{1}{2} \mathrm{~m}_{41}^{2}-\frac{1}{2} \mathrm{~m}_{42}^{2}
$$

When $d_{41}$ and $d_{42}$ are indeed small, such as this case, then $m_{43}$ is negligible with respect to $m_{41}$ and $\mathrm{m}_{42}$. Then using Eqs. (12) we can write

$$
\left[\begin{array}{l}
\mathrm{m}_{41} \\
\mathrm{~m}_{42} \\
\mathrm{~m}_{43}
\end{array}\right]=\left[\begin{array}{ll}
1 & 0 \\
0 & 1 \\
0 & 0
\end{array}\right]\left[\begin{array}{l}
\mathrm{d}_{41} \\
\mathrm{~d}_{42}
\end{array}\right]
$$

It is the choice to express the component of $\mathrm{m}_{\mathrm{j}}$ along the gyro sensitive axis (in this case $\mathrm{m}_{43}$ ) that enables its elimination.

For gyros whose sensitive axes are not aligned along one of the body axes the computation is more elaborate. Consider for example $G_{2}$, the second gyro of the EOS-AQUA satellite. In order to define its misalignment in the body coordinates let us define a coordinate system in which the gyro sensitive axis is nominally aligned along one of its axes. Such a system (X", Y", Z") is presented in Fig. 3, where the sensitive axis of the $G_{2}$ gyro is aligned along the system $Y$ " axis. Following the preceding development for the $\mathrm{G}_{4}$ gyro we conclude that

$$
\mathrm{m}_{21}^{\prime \prime}=\mathrm{d}_{21}^{\prime \prime} \quad \text { (16.a) } \quad \mathrm{m}_{23}^{\prime \prime}=\mathrm{d}_{23}^{\prime \prime}
$$




$$
\mathrm{m}_{22}^{\prime \prime}=-\frac{1}{2} \mathrm{~d}_{21}^{\prime 2}-\frac{1}{2} \mathrm{~d}_{23}^{\prime 2}=-\frac{1}{2} \mathrm{~m}_{21}^{\prime 2}-\frac{1}{2} \mathrm{~m}_{23}^{\prime 2}
$$

(The " sign denotes the fact that the values are expressed in the X", Y", Z" coordinate system.) Here too, the misalignment along the sensitive axis, $\mathrm{m}_{22}^{\prime \prime}$, is normally negligible. In order to compute the misalignment error in the gyro reading we have to use Eq. (9) where the angular rate vector is transformed to the double prime coordinate system and the misalignment parameters are those given in Eqs. (16.a and b). As shown in Fig. 3 the transformation from the body to the double prime coordinates is performed by
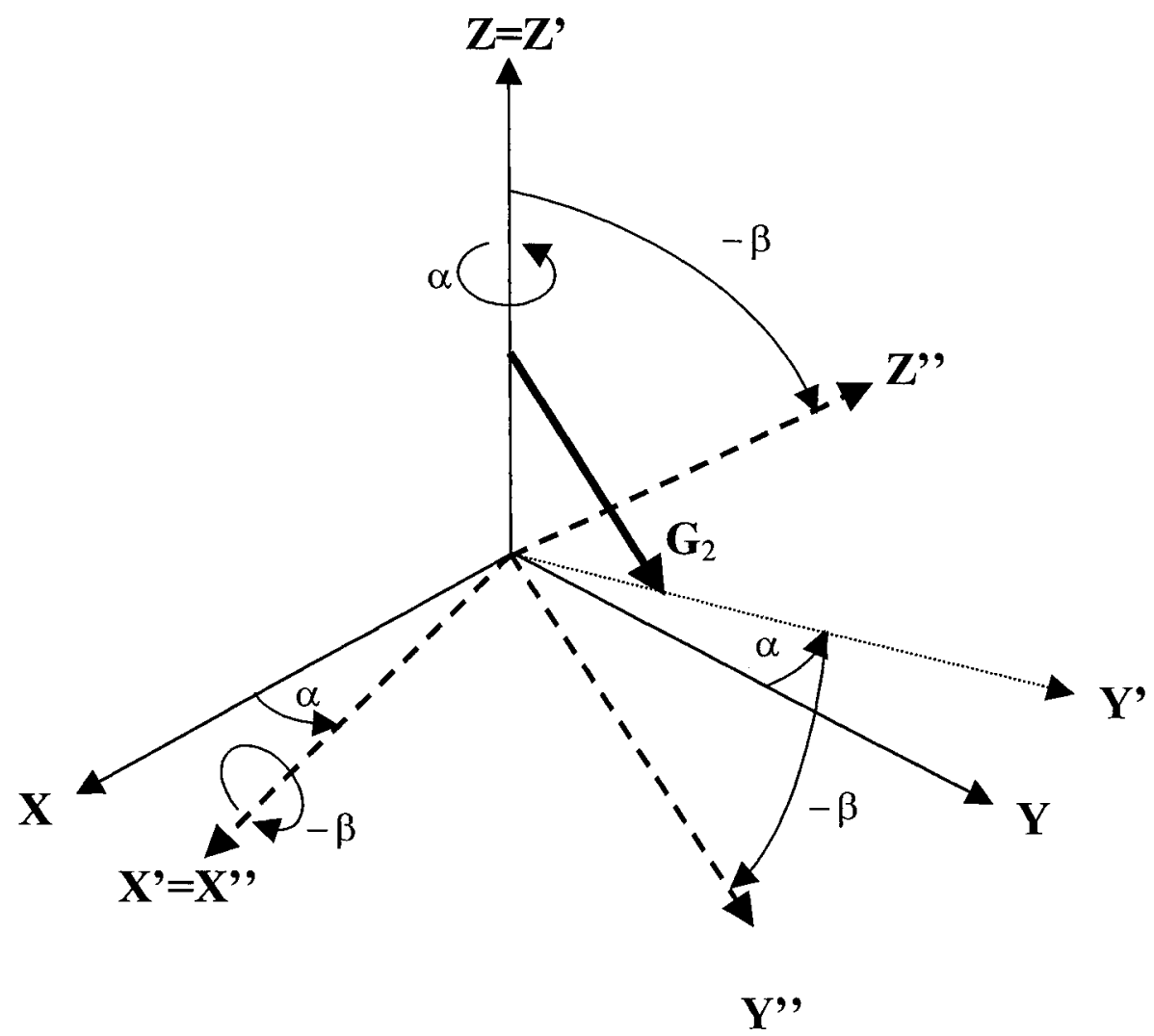

Fig. 3: The Transformation from the $\mathrm{G}_{2}$ Body to Gyro Coordinates.

two rotations. The first rotation is by an angle $\alpha$ about the $\mathbf{Z}$ axis, and the second is by an angle $-\beta$ about the $\mathbf{X}$ ' axis. The resulting transformation matrix from the body to the $\mathrm{G}_{2}$ coordinates is therefore

and

$$
R_{2}^{b}=\left[\begin{array}{ccc}
c \alpha & s \alpha & 0 \\
-s \alpha \cdot c \beta & c \alpha \cdot c \beta & -s \beta \\
-s \alpha \cdot s \beta & c \alpha \cdot s \beta & c \beta
\end{array}\right]
$$

then following Eq. (9)

$$
\omega_{2}=R_{2}^{b} \omega_{b}
$$




$$
\Delta \mathrm{G}_{2}^{\mathrm{m}}=\left[\mathrm{R}_{2}^{\mathrm{b}} \boldsymbol{\omega}_{\mathrm{b}}\right]^{\mathrm{T}} \mathbf{d}_{2^{*}}^{\prime \prime}=\boldsymbol{\omega}_{\mathrm{b}}^{\mathrm{T}} \mathrm{R}_{\mathrm{b}}^{2} \mathbf{d}_{2^{*}}^{\prime \prime}
$$

where

$$
\left[\mathbf{d}_{2^{*}}^{\prime \prime}\right]^{\mathrm{T}}=\left[\begin{array}{lll}
\mathrm{d}_{21}^{\prime \prime} & 0 & \mathrm{~d}_{23}^{\prime \prime}
\end{array}\right]
$$

It is easy to see that

$$
\mathrm{R}_{\mathrm{b}}^{2} \mathbf{d}_{2^{*}}^{\prime \prime}=\left[\begin{array}{cc}
\mathrm{c} \alpha & -\mathrm{s} \alpha \cdot \mathrm{s} \beta \\
\mathrm{s} \alpha & \mathrm{c} \alpha \cdot \mathrm{s} \beta \\
0 & \mathrm{c} \beta
\end{array}\right]\left[\begin{array}{l}
\mathrm{d}_{2 \mathrm{t}}^{\prime \prime} \\
\mathrm{d}_{23}^{\prime \prime}
\end{array}\right]
$$

Define

$$
\mathrm{E}_{2}=\left[\begin{array}{cc}
\mathrm{c} \alpha & -\mathrm{s} \alpha \cdot \mathrm{s} \beta \\
\mathrm{s} \alpha & \mathrm{c} \alpha \cdot \mathrm{s} \beta \\
0 & \mathrm{c} \beta
\end{array}\right] \quad \text { (23.a) } \quad \mathbf{d}_{2}=\left[\begin{array}{l}
\mathrm{d}_{21}^{\prime \prime} \\
\mathrm{d}_{23}^{\prime \prime}
\end{array}\right]
$$

then Eq. (20) can be written as

$$
\Delta \mathrm{G}_{2}^{\mathrm{m}}=\boldsymbol{\omega}_{\mathrm{b}}^{\mathrm{T}} \mathrm{E}_{2} \mathbf{d}_{2}
$$

To evaluate $E_{2}$, we need to compute the angles $\alpha$ and $\beta$. For this we turn to Fig. 4 where these angles

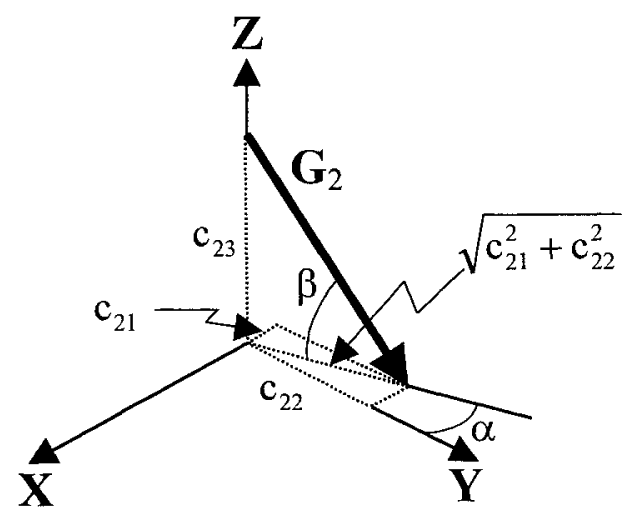

Fig. 4: Definition of the Rotation Angles $\alpha$ and $\beta$.

are defined in the projection of the $\mathrm{G}_{2}$ direction on the body axes. From this figure we conclude that

$$
\begin{aligned}
& \alpha=\tan ^{-1}\left(\left|\frac{c_{21}}{c_{22}}\right|\right) \\
& \beta=\cos ^{-1}\left(\frac{\sqrt{c_{21}^{2}+c_{22}^{2}}}{1}\right)=\cos ^{-1}\left(\sqrt{c_{21}^{2}+c_{22}^{2}}\right)
\end{aligned}
$$

Using the EOS-AQUA satellite values (see Eqs. (4) and (5)) we obtain 


$$
\begin{aligned}
& \alpha=\tan ^{-1}\left(\left|\frac{c_{21}}{c_{22}}\right|\right)=\tan ^{-1}\left(\frac{\sqrt{\frac{1}{6}}}{\sqrt{\frac{1}{2}}}\right)=\tan ^{-1}\left(\frac{1}{\sqrt{3}}\right)=30^{\circ} \\
& \beta=\cos ^{-1}\left(\sqrt{c_{21}^{2}+c_{22}^{2}}\right)=\cos ^{-1}\left(\sqrt{\frac{1}{6}+\frac{1}{2}}\right)=\cos ^{-1}\left(\sqrt{\frac{2}{3}}\right)=35.26^{\circ}
\end{aligned}
$$

Due to the symmetry between the positioning of the $G_{2}$ and the $G_{3}$ gyros, it is easy to see that when considering the $G_{3}$, the first rotation is about the new $\mathbf{Z}$ axis by the angle $\pi-\alpha$ and then about the $\mathbf{X}$, axis by the angle $-\beta$. Therefore

$$
R_{3}^{b}=\left[\begin{array}{ccc}
-c \alpha & s \alpha & 0 \\
-s \alpha \cdot c \beta & -c \alpha \cdot c \beta & -s \beta \\
-s \alpha \cdot s \beta & -c \alpha \cdot s \beta & c \beta
\end{array}\right]
$$

and similarly to Eq. (19) for this transformation we obtain

then following Eq. (20)

$$
\omega_{3}=\mathrm{R}_{3}^{\mathrm{b}} \boldsymbol{\omega}_{\mathrm{b}}
$$

$$
\Delta \mathrm{G}_{3}^{\mathrm{m}}=\left[\mathrm{R}_{3}^{\mathrm{b}} \boldsymbol{\omega}_{\mathrm{b}}\right]^{\mathrm{T}} \mathrm{d}_{3^{*}}^{\prime \prime}=\omega_{\mathrm{b}}^{\mathrm{T}} \mathrm{R}_{\mathrm{b}}^{3} \mathrm{~d}_{3^{*}}
$$

where

It is easy to see that

$$
\left[\mathbf{d}_{3^{*}}\right]^{\mathrm{T}}=\left[\begin{array}{lll}
\mathrm{d}_{31}^{\prime \prime} & 0 & \mathrm{~d}_{33}^{\prime \prime}
\end{array}\right]
$$

$$
\mathrm{R}_{\mathrm{b}}^{3} \mathbf{d}_{3^{*}}^{\prime \prime}=\left[\begin{array}{cc}
-\mathrm{c} \alpha & -\mathrm{s} \alpha \cdot \mathrm{s} \beta \\
\mathrm{s} \alpha & -\mathrm{c} \alpha \cdot \mathrm{s} \beta \\
0 & \mathrm{c} \beta
\end{array}\right]\left[\begin{array}{l}
\mathrm{d}_{31}^{\prime \prime} \\
\mathrm{d}_{33}^{\prime \prime}
\end{array}\right]
$$

Define

$$
E_{3}=\left[\begin{array}{cc}
-c \alpha & -s \alpha \cdot s \beta \\
s \alpha & -c \alpha \cdot s \beta \\
0 & c \beta
\end{array}\right] \quad \text { (31.a) } \quad \mathbf{d}_{3}=\left[\begin{array}{l}
d_{31}^{\prime \prime} \\
d_{33}^{\prime \prime}
\end{array}\right]
$$

then Eq. (28) can be written as

$$
\Delta \mathrm{G}_{3}^{\mathrm{m}}=\boldsymbol{\omega}_{\mathrm{b}}^{\mathrm{T}} \mathrm{E}_{3} \mathbf{d}_{3}
$$

For the $G_{1}$ gyro we have only one transformation, which brings the body $\mathbf{X}$-axis into coincidence with the $G_{1}$ gyro sensitive axis (see Fig. 2). It is about the $Y$-axis by an angle which we denote by $\gamma$. For this gyro we have then

$$
R_{1}^{b}=\left[\begin{array}{ccc}
c \gamma & 0 & -s \gamma \\
0 & 1 & 0 \\
s \gamma & 0 & c \gamma
\end{array}\right]
$$

and the angular rate in coordinate system 1 is then 


$$
\omega_{1}=\mathrm{R}_{1}^{\mathrm{b}} \omega_{\mathrm{b}}
$$

and

$$
\Delta \mathrm{G}_{1}^{\mathrm{m}}=\left[\mathrm{R}_{1}^{\mathrm{b}} \boldsymbol{\omega}_{\mathrm{b}}\right]^{\mathrm{T}} \mathbf{d}_{1^{*}}^{\prime \prime}=\boldsymbol{\omega}_{\mathrm{b}}^{\mathrm{T}} \mathrm{R}_{\mathrm{b}}^{1} \mathbf{d}_{1^{*}}^{\prime}
$$

where

$$
\left[\mathbf{d}_{1^{*}}^{\prime}\right]^{\mathrm{T}}=\left[\begin{array}{lll}
0 & \mathrm{~d}_{12}^{\prime} & \mathrm{d}_{23}^{\prime}
\end{array}\right]
$$

We denote the misalignment parameters of this gyro by a single prime because it takes only one rotation (to a single prime system) to align the coordinate axis with the sensitive axis of the $G_{1}$ gyro. Note that here the misalignments that are not negligible are $d_{12}^{\prime}$ and $d_{13}^{\prime}$. It is easy to see that

$$
\mathbf{R}_{b}^{1} \mathbf{d}_{1^{*}}^{\prime \prime}=\left[\begin{array}{cc}
0 & \mathbf{s} \gamma \\
1 & 0 \\
0 & \mathrm{c} \gamma
\end{array}\right]\left[\begin{array}{l}
\mathrm{d}_{12}^{\prime} \\
\mathrm{d}_{13}^{\prime}
\end{array}\right]
$$

Define

$$
E_{1}=\left[\begin{array}{cc}
0 & s \gamma \\
1 & 0 \\
0 & c \gamma
\end{array}\right] \quad(38 . a) \quad \mathbf{d}_{1}=\left[\begin{array}{l}
d_{12}^{\prime \prime} \\
d_{13}^{\prime \prime}
\end{array}\right]
$$

then Eq. (35) can be written as

$$
\Delta \mathrm{G}_{1}^{\mathrm{m}}=\boldsymbol{\omega}_{\mathrm{b}}^{\mathrm{T}} \mathrm{E}_{1} \mathbf{d}_{1}
$$

From fig. 2 it is easy to see that the rotation angle, $\gamma$, is computable, as follows;

$$
\gamma=\sin ^{-1}\left(\frac{\left|\mathbf{c}_{13}\right|}{1}\right)=\sin ^{-1}\left(\sqrt{\frac{1}{3}}\right)=35.26^{\circ}
$$

Similarly to the computations carried out for the misalignment errors for gyros 1,2 , and 3 , we can write for gyro 4

$$
\Delta \mathrm{G}_{4}^{\mathrm{m}}=\boldsymbol{\omega}_{\mathrm{b}}^{\mathrm{T}} \mathrm{E}_{4} \mathbf{d}_{4}
$$

where, based on Eq. (16),

$$
\mathrm{E}_{4}=\left[\begin{array}{ll}
1 & 0 \\
0 & 1 \\
0 & 0
\end{array}\right](42 . \mathrm{a}) \quad \mathbf{d}_{4}=\left[\begin{array}{l}
\mathrm{d}_{41} \\
\mathrm{~d}_{42}
\end{array}\right]
$$

Let

$$
\begin{aligned}
& {\left[\Delta \mathbf{G}^{\mathrm{m}}\right]^{\mathrm{T}}=\left[\begin{array}{llll}
\Delta \mathrm{G}_{1}^{\mathrm{m}} & \Delta \mathrm{G}_{2}^{\mathrm{m}} & \Delta \mathrm{G}_{3}^{\mathrm{m}} & \Delta \mathrm{G}_{4}^{\mathrm{m}}
\end{array}\right]} \\
& \Omega^{\mathrm{m}}=\left[\begin{array}{cccccccccccc}
\omega_{\mathrm{x}} & \omega_{\mathrm{y}} & \omega_{\mathrm{z}} & 0 & 0 & 0 & 0 & 0 & 0 & 0 & 0 & 0 \\
0 & 0 & 0 & \omega_{\mathrm{x}} & \omega_{\mathrm{y}} & \omega_{\mathrm{z}} & 0 & 0 & 0 & 0 & 0 & 0 \\
0 & 0 & 0 & 0 & 0 & 0 & \omega_{\mathrm{x}} & \omega_{\mathrm{y}} & \omega_{\mathrm{z}} & 0 & 0 & 0 \\
0 & 0 & 0 & 0 & 0 & 0 & 0 & 0 & 0 & \omega_{\mathrm{x}} & \omega_{\mathrm{y}} & \omega_{\mathrm{z}}
\end{array}\right]
\end{aligned}
$$




$$
\mathrm{E}=\left[\begin{array}{cccc}
\mathrm{E}_{1} & 0 & 0 & 0 \\
0 & \mathrm{E}_{2} & 0 & 0 \\
0 & 0 & \mathrm{E}_{3} & 0 \\
0 & 0 & 0 & \mathrm{E}_{4}
\end{array}\right] \quad(43 . c) \quad \mathbf{d}^{\mathrm{T}}=\left[\begin{array}{llll}
\mathbf{d}_{1}^{\mathrm{T}} & \mathbf{d}_{2}^{\mathrm{T}} & \mathbf{d}_{3}^{\mathrm{T}} & \mathbf{d}_{4}^{\mathrm{T}}
\end{array}\right]
$$

Then Eqs. (39), (23), (32) and (41) can be unified into the following single equation

$$
\Delta \mathbf{G}^{\mathrm{m}}=\Omega^{\mathrm{m}} \mathrm{E} \mathbf{d}
$$

\section{Scale Factor Error Model}

As mentioned, another error source that causes the difference between the correct value of the rates and their measurements are the scale factor errors. The error model for the scale factor error is simply

$$
\Delta \mathbf{G}^{\mathrm{k}}=\left[\begin{array}{c}
\omega_{\mathrm{G} 1} \mathrm{k}_{1} \\
\omega_{\mathrm{G} 2} \mathrm{k}_{2} \\
\omega_{\mathrm{G} 3} \mathrm{k}_{3} \\
\omega_{\mathrm{G} 4} \mathrm{k}_{4}
\end{array}\right]
$$

where the subscript $k$ denotes the fact that this error is caused by gyro scale factor error, $\omega_{\mathrm{Gi}}, i=1-4$ is the angular velocity measured by gyro number $i$, and $\mathrm{k}_{\mathrm{i}}$ is the scale factor error of that gyro. The actual components $\omega_{\mathrm{Gi}}$ are obtained by transforming the angular velocity expressed in body coordinates to the actual misaligned gyro sensitive axes using the matrix D; however, because $\mathrm{D}$ is unknown to us we use instead the matrix $\mathrm{C}$ that transforms the body rate to the nominal gyro axes, and is close enough to $\mathrm{D}$. Thus,

$$
\left[\begin{array}{c}
\omega_{\mathrm{G} 1} \\
\omega_{\mathrm{G} 2} \\
\omega_{\mathrm{G} 3} \\
\omega_{\mathrm{G} 4}
\end{array}\right]=\mathrm{C}\left[\begin{array}{l}
\omega_{\mathrm{x}} \\
\omega_{\mathrm{y}} \\
\omega_{\mathrm{z}}
\end{array}\right]
$$

where in the case of EOS-AQUA, C is as given in Eq. (5). Equation (45) can be written as follows

Define

$$
\Delta \mathbf{G}^{\mathrm{k}}=\left[\begin{array}{cccc}
\omega_{\mathrm{G} 1} & 0 & 0 & 0 \\
0 & \omega_{\mathrm{G} 2} & 0 & 0 \\
0 & 0 & \omega_{\mathrm{G} 3} & 0 \\
0 & 0 & 0 & \omega_{\mathrm{G} 4}
\end{array}\right]\left[\begin{array}{l}
\mathrm{k}_{1} \\
\mathrm{k}_{2} \\
\mathrm{k}_{3} \\
\mathrm{k}_{4}
\end{array}\right]
$$

$$
\Omega^{\mathrm{k}}=\left[\begin{array}{cccc}
\omega_{\mathrm{G} 1} & 0 & 0 & 0 \\
0 & \omega_{\mathrm{G} 2} & 0 & 0 \\
0 & 0 & \omega_{\mathrm{G} 3} & 0 \\
0 & 0 & 0 & \omega_{\mathrm{G} 4}
\end{array}\right]
$$


and

then Eq. (47) can be written as

$$
\mathbf{k}^{\mathrm{T}}=\left[\begin{array}{llll}
\mathrm{k}_{1} & \mathrm{k}_{2} & \mathrm{k}_{3} & \mathrm{k}_{4}
\end{array}\right]
$$

$$
\Delta \mathbf{G}^{\mathrm{k}}=\Omega^{\mathrm{k}} \mathbf{k}
$$

\section{Bias Model}

The bias error model is quite simple and is given by

$$
\mathbf{b}=\left[\begin{array}{l}
b_{1} \\
b_{2} \\
b_{3} \\
b_{4}
\end{array}\right]
$$

where $b_{\mathrm{i}}$ is the bias of gyro number $i$.

\section{The Augmented Gyro Error Model}

The total gyro error is the sum of all the errors discussed before; namely, bias, scale factor and misalignment errors; that is

or using Eqs. (44) and (48.c)

$$
\begin{aligned}
& \Delta \mathbf{G}=\Delta \mathbf{G}^{\mathrm{m}}+\Delta \mathbf{G}^{\mathrm{k}}+\mathbf{b} \\
& \Delta \mathbf{G}=\Omega^{\mathrm{m}} \mathrm{E} \mathbf{d}+\Omega^{\mathrm{k}} \mathbf{k}+\mathbf{b}
\end{aligned}
$$

The last equation can be written in the following form

$$
\mathbf{G}_{\mathrm{a}}-\mathrm{C} \boldsymbol{\omega}_{\mathrm{r}}=\left[\begin{array}{lll}
\Omega^{\mathrm{m}} \mathrm{E} & \Omega^{\mathrm{k}} & \mathrm{I}_{4}
\end{array}\right]\left[\begin{array}{l}
\mathbf{d} \\
\mathbf{k} \\
\mathbf{b}
\end{array}\right]
$$

where $\omega_{\mathrm{r}}$ is the reference angular velocity vector. It is the angular velocity, which the SC experiences in reality. As mentioned before, $\mathbf{G}_{\mathrm{n}}$ is the nominal angular velocity measured by the four gyros. The vector of the left hand side of the last equation as well as the matrix on the right hand side are functions of the body angular rate, $\boldsymbol{\omega}_{\mathrm{r}}$. We denote them as follows

also let

$$
\begin{gathered}
\mathbf{y}\left(\boldsymbol{\omega}_{\mathrm{r}}\right)=\mathbf{G}_{\mathrm{a}}-\mathrm{C} \boldsymbol{\omega}_{\mathrm{r}} \\
\mathrm{H}\left(\boldsymbol{\omega}_{\mathrm{r}}\right)=\left[\begin{array}{lll}
\Omega^{\mathrm{m}} \mathrm{E} & \Omega^{\mathrm{k}} & \mathrm{I}_{4}
\end{array}\right]
\end{gathered}
$$

$$
\mathbf{x}=\left[\begin{array}{l}
\mathbf{d} \\
\mathbf{k} \\
\mathbf{b}
\end{array}\right]
$$

then Eq. (50.c) can be written as

$$
\mathbf{y}\left(\boldsymbol{\omega}_{\mathrm{r}}\right)=\mathrm{H}\left(\boldsymbol{\omega}_{\mathrm{r}}\right) \mathbf{x}
$$




\section{CALIBRATION-PARAMETERS ESTIMATION FOR KNOWN RATE}

Our goal now is to estimate $\mathbf{x}$, and for that we need to know the angular rate, which the gyros are set to measure. We distinguish between two major cases. One case is that where $\boldsymbol{\omega}_{\mathrm{r}}$, the reference SC angular velocity, is known, and the other case is that where the rate is not known and has to be evaluated simultaneously with the estimate of $\mathbf{x}$. In the first case we also distinguish between the deterministic and stochastic cases. All these cases are discussed next.

\section{Deterministic Case}

When the SC rotates at a certain angular rate and a one-time measurement of the four gyro readings is taken at that time, which we denote by $t_{k}$, we obtain one matrix equation, as follows:

$$
\mathbf{y}\left(\boldsymbol{\omega}_{\mathrm{r}, \mathrm{k}}\right)=\mathrm{H}\left(\boldsymbol{\omega}_{\mathrm{r}, \mathrm{k}}\right) \mathbf{x}
$$

where $\boldsymbol{\omega}_{\mathrm{r}, \mathrm{k}}$ denotes the angular rate at time $t_{k}$. This yields four equations for the 16 unknowns of $\mathbf{x}$. If the rate does not change, then more measurements do not change the equations. A change in the angular rate of the SC is needed to generate more equations. It should be noted that even if we have 16 equations, it does not mean that they are all independent and that we can solve for $\mathbf{x}$. We have to design the profile of $\omega_{\mathrm{r}}$ and the times when measurements are taken in such a way that we will be able to find 16 independent equations. Let us denote the 16 independent equations by one matrix equation, as follows:

$$
\widetilde{\mathbf{y}}=\widetilde{\mathrm{H}} \mathbf{x}
$$

Because we have 16 independent equations, $\widetilde{\mathrm{H}}$ has an inverse; therefore, we can solve for $\mathbf{x}$ using

$$
\mathbf{x}=\widetilde{\mathrm{H}}^{-1} \widetilde{\mathbf{y}}
$$

\section{Stochastic Case}

In this case we assume that the measurements are contaminated by noise, which is the most likely case. Therefore, the matrix equation that describes this case at time $t_{k}$ is

$$
\mathbf{y}\left(\boldsymbol{\omega}_{\mathrm{r}, \mathrm{k}}\right)=\mathrm{H}\left(\boldsymbol{\omega}_{\mathrm{r}, \mathrm{k}}\right) \mathbf{x}+\mathbf{v}_{\mathrm{k}}
$$

Even if we find 16 independent equations from measurements done at different time points we still want to use all available measurements and obtain $\mathbf{x}$ as a least squares estimate. We have

$$
\left[\begin{array}{l}
\mathbf{y}\left(\boldsymbol{\omega}_{\mathrm{r}, 1}\right) \\
\mathbf{y}\left(\boldsymbol{\omega}_{\mathrm{r}, 2}\right) \\
\mathbf{y}\left(\boldsymbol{\omega}_{\mathrm{r}, 3}\right) \\
\mathbf{y}\left(\boldsymbol{\omega}_{\mathrm{r}, 4}\right)
\end{array}\right]=\left[\begin{array}{l}
\mathrm{H}\left(\boldsymbol{\omega}_{\mathrm{r}, 1}\right) \\
\mathrm{H}\left(\boldsymbol{\omega}_{\mathrm{r}, 2}\right) \\
\mathrm{H}\left(\boldsymbol{\omega}_{\mathrm{r}, 3}\right) \\
\mathrm{H}\left(\boldsymbol{\omega}_{\mathrm{r}, 4}\right)
\end{array}\right] \mathbf{x}+\left[\begin{array}{l}
\mathbf{v}_{1} \\
\mathbf{v}_{2} \\
\mathbf{v}_{3} \\
\mathbf{v}_{4}
\end{array}\right]
$$

Let 


$$
\mathbf{Y}=\left[\begin{array}{l}
\mathbf{y}\left(\omega_{\mathrm{r}, 1}\right) \\
\mathbf{y}\left(\boldsymbol{\omega}_{\mathrm{r}, 2}\right) \\
\mathbf{y}\left(\boldsymbol{\omega}_{\mathrm{r}, 3}\right) \\
\mathbf{y}\left(\boldsymbol{\omega}_{\mathrm{r}, 4}\right)
\end{array}\right] \quad(57 . \mathrm{a}) \quad \mathbf{H}=\left[\begin{array}{l}
\mathrm{H}\left(\boldsymbol{\omega}_{\mathrm{r}, 1}\right) \\
\mathrm{H}\left(\boldsymbol{\omega}_{\mathrm{r}, 2}\right) \\
\mathrm{H}\left(\boldsymbol{\omega}_{\mathrm{r}, 3}\right) \\
\mathrm{H}\left(\boldsymbol{\omega}_{\mathrm{r}, 4}\right)
\end{array}\right]
$$

then $\hat{\mathbf{x}}$, the least squares fit to $\mathbf{x}$, is as follows (ref. 5)

$$
\hat{\mathbf{x}}=\left(\mathrm{H}^{\mathrm{T}} \mathrm{H}\right)^{-1} \mathrm{H}^{\mathrm{T}} \mathbf{Y}
$$

The profile of $\omega_{\mathrm{r}}$ has to be chosen in a careful way as to enhance the observability of $\mathbf{X}$.

\section{CALIBRATION-PARAMETERS ESTIMATION FOR UNKNOWN RATE}

In this case we have to find the angular rate vector while estimating the calibration parameters. The information that we have is attitude information and gyro measurements. We need the attitude information in order to estimate the angular rate, and we need the gyro measurements, as well as the estimated angular rate, for the calibration process. The attitude information can be supplied in various ways; namely, we may have it in the form of raw vector measurements or we can have it in an already processed form as attitude quaternion for example. The angular rate behaves according to the following $\mathrm{SC}$ angular dynamics equation

$$
\dot{\omega}=\mathrm{I}^{-1}[(\mathrm{I} \boldsymbol{\omega}+\mathbf{h}) \times] \omega+\mathrm{I}^{-1}(\mathbf{T}-\dot{\mathbf{h}})
$$

where I is the SC inertia tensor, $[(\mathrm{I} \boldsymbol{\omega}+\mathbf{h}) \times]$ is the cross product matrix of the vector $(\mathrm{I} \boldsymbol{\omega}+\mathbf{h}), \mathbf{h}$ is the angular momentum of the momentum wheels, and $\mathbf{T}$ is the external torque operating on the SC. Because $\mathbf{x}$ is a constant vector, it obeys the following differential equation

$$
\dot{\mathbf{x}}=0
$$

We are tempted to combine the last two equations into one dynamics equation, as follows;

$$
\left[\begin{array}{c}
\dot{\boldsymbol{\omega}} \\
\dot{\mathbf{x}}
\end{array}\right]=\left[\begin{array}{cc}
\mathrm{I}^{-1}[(\mathrm{I} \boldsymbol{\omega}+\mathbf{h}) \times] & 0 \\
0 & 0
\end{array}\right]\left[\begin{array}{l}
\boldsymbol{\omega} \\
\mathbf{x}
\end{array}\right]+\left[\begin{array}{c}
\mathrm{I}^{-1}(\mathbf{T}-\dot{\mathbf{h}}) \\
0
\end{array}\right]
$$

This dynamics model calls for the use of a Kalman Filter (KF). In fact the most appropriate filter is the Pseudo-Linear Kalman (PSELIKA, ref. 6) filter. To find the suitable measurement equation, we turn to Eqs. (50.c) and (51.b) from which it is obvious that

$$
\mathbf{G}_{\mathrm{a}}=\left[\begin{array}{ll}
\mathrm{C} & \mathrm{H}(\boldsymbol{\omega})
\end{array}\right]\left[\begin{array}{l}
\boldsymbol{\omega} \\
\mathbf{x}
\end{array}\right]
$$

In order to apply the filter algorithm we need to add some process noise to Eq. (59.c) and some measurement noise to Eq. (60). However, it is easy to see though that the last system is unobservable. Theoretically, if the actual noise values are small, and if we know the initial angular velocity, then we can compute the angular velocity separately. Once we know the angular rate and we command the SC to execute a suitable angular rate profile, we should be able to compute the calibration parameters. In reality though the computation of the angular rate is not accurate enough because it is done in an open loop manner. Therefore, we have to add attitude measurements in order to check the divergence of the computed angular rate. In this case we can indeed combine all the dynamics equations into one augmented matrix 
equation and estimate the augmented state vector. This is so because it is possible to find an angular rate profile that will render the system observable.

As mentioned, attitude can be given in several ways; namely, it can be given in a raw form as vector measurements or in processed attitude parameters like a quaternion or direction cosine matrix (DCM). Let us consider two cases, one where attitude is represented by a processed quaternion, and the other case when we have raw vector measurements. The case where attitude is given in the form of a DCM can be inferred from the development presented in Ref. 7 and the way we handle quaternion representation of attitude.

\section{Estimation When Attitude is Presented by the Attitude Quaternion}

Let us assume first that the attitude is given in a form of a quaternion (ref. 8). In this case the filter dynamics is as follows (ref. 7)

$$
\left[\begin{array}{c}
\dot{\boldsymbol{\omega}} \\
\dot{\mathbf{x}} \\
\dot{\mathbf{q}}
\end{array}\right]=\left[\begin{array}{ccc}
\mathrm{I}^{-1}[(\mathrm{I} \boldsymbol{\omega}+\mathbf{h}) \times] & 0 & 0 \\
0 & 0 & 0 \\
\frac{1}{2} \mathrm{Q} & 0 & 0
\end{array}\right]\left[\begin{array}{l}
\boldsymbol{\omega} \\
\mathbf{x} \\
\mathbf{q}
\end{array}\right]+\left[\begin{array}{c}
\mathrm{I}^{-1}(\mathbf{T}-\dot{\mathbf{h}}) \\
0 \\
0
\end{array}\right]
$$

where

$$
Q=\left[\begin{array}{ccc}
q_{4} & -q_{3} & q_{2} \\
q_{3} & q_{4} & -q_{1} \\
-q_{2} & q_{1} & q_{4} \\
-q_{1} & -q_{2} & -q_{3}
\end{array}\right]
$$

and the corresponding measurement equation is

$$
\mathbf{q}_{\mathrm{m}}=\left[\begin{array}{lll}
0_{4 \times 3} & 0_{4 \times 16} & \mathrm{I}_{4 \times 4}
\end{array}\right]\left[\begin{array}{l}
\boldsymbol{\omega} \\
\mathbf{x} \\
\mathbf{q}
\end{array}\right]
$$

The matrix $\mathrm{I}_{4 \times 4}$ is a fourth order identity matrix. The combined measurement equation consists of Eqs. (60) and (63); that is

$$
\left[\begin{array}{l}
\mathbf{G}_{\mathrm{a}} \\
\mathbf{q}_{\mathrm{m}}
\end{array}\right]=\left[\begin{array}{ccc}
\mathrm{C} & \mathrm{H}(\boldsymbol{\omega}) & 0_{4 \times 4} \\
0_{4 \times 3} & 0_{4 \times 16} & \mathrm{I}_{4 \times 4}
\end{array}\right]\left[\begin{array}{c}
\boldsymbol{\omega} \\
\mathbf{x} \\
\mathbf{q}
\end{array}\right]
$$

\section{Estimation When Attitude is Given by Vector Observations}

Normally, in space missions attitude is determined from vector observations. These observations can be used directly to check the divergence of the angular velocity estimates (ref .7). This is shown next. Suppose that we have $\mathrm{N}$ vector measurements at a certain time point. Let $\mathbf{r}_{\mathrm{i}}$ denote some abstract $\mathrm{i}^{\text {th }}$ vector as expressed in the reference coordinate system, and let $\mathbf{b}_{\mathrm{i}}$ denote the same vector when expressed in the body coordinates. From the laws of dynamics it is known that

$$
\mathrm{D} \dot{\mathbf{r}}_{\mathrm{i}}=\dot{\mathbf{b}}_{\mathrm{i}}+\boldsymbol{\omega} \times \mathbf{b}_{\mathrm{i}}
$$


where $\dot{\mathbf{r}}_{\mathrm{i}}$ is the time derivative of $\mathbf{r}_{\mathrm{i}}$ as seen by an observer in the reference coordinates, $\mathrm{D}$ is the matrix that transforms vectors from the reference to body coordinates, and $\dot{\mathbf{b}}_{\mathrm{i}}$ is the time derivative of $\mathbf{b}_{\mathrm{i}}$ as seen by an observer in body coordinates. The vector $\mathbf{b}_{i}$ is a measured vector and $\dot{\mathbf{b}}_{\mathrm{i}}$ is its time derivative. We can write Eq. (65), as follows;

$$
\dot{\mathbf{b}}_{\mathrm{i}}=\left[\mathbf{b}_{\mathrm{i}} \times\right] \mathbf{\omega}+\mathrm{D} \dot{\mathbf{r}}_{\mathrm{i}}
$$

Note that $\dot{\mathbf{r}}_{i}$ is computable since $\mathbf{r}_{i}$ is usually known because, generally, the vector is a direction to a certain known planet whose location is given in an Almanac or, like with magnetometer measurements, the vector can be computed using a model. (It should be noted that quite often the rate of change of $\mathbf{r}_{i}$ is so small that $\dot{\mathbf{r}}_{\mathrm{i}}$ is negligible). Define

$$
\dot{\boldsymbol{\beta}}=\left[\begin{array}{c}
\dot{\mathbf{b}}_{1} \\
\cdot \\
\cdot \\
\dot{\mathbf{b}}_{\mathrm{N}}
\end{array}\right] \quad(67 . \mathrm{a}) \quad \mathrm{B}=\left[\begin{array}{c}
{\left[\mathbf{b}_{1} \times\right]} \\
\cdot \\
\cdot \\
{\left[\mathbf{b}_{\mathrm{N}} \times\right]}
\end{array}\right] \quad(67 . \mathbf{b}) \text { and } \mathbf{u}=\left[\begin{array}{c}
\mathrm{D} \dot{\mathbf{r}}_{1} \\
\cdot \\
\cdot \\
\mathrm{D} \dot{\mathbf{r}}_{\mathrm{N}}
\end{array}\right]
$$

then we can augment all the $\mathrm{N}$ equations of Eq. (66) into one matrix equation, as follows;

$$
\dot{\boldsymbol{\beta}}=\mathrm{B} \omega+\mathbf{u}
$$

therefore, instead of Eq. (61), we obtain in this case of vector measurement the augmented equation

$$
\left[\begin{array}{c}
\dot{\boldsymbol{\omega}} \\
\dot{\mathbf{x}} \\
\dot{\boldsymbol{\beta}}
\end{array}\right]=\left[\begin{array}{ccc}
\mathrm{I}^{-1}[(\mathrm{I} \boldsymbol{\omega}+\mathbf{h}) \times] & 0 & 0 \\
0 & 0 & 0 \\
\mathrm{~B} & 0 & 0
\end{array}\right]\left[\begin{array}{c}
\boldsymbol{\omega} \\
\mathbf{x} \\
\boldsymbol{\beta}
\end{array}\right]+\left[\begin{array}{c}
\mathrm{I}^{-1}(\mathbf{T}-\dot{\mathbf{h}}) \\
0 \\
\mathbf{u}
\end{array}\right]
$$

and the corresponding measurement equation is

$$
\boldsymbol{\beta}_{\mathrm{m}}=\left[\begin{array}{lll}
0_{3 \times 3} & 0_{3 \times 16} & \mathrm{I}_{3 \times 3}
\end{array}\right]\left[\begin{array}{c}
\boldsymbol{\omega} \\
\mathbf{x} \\
\boldsymbol{\beta}
\end{array}\right]
$$

whereas the augmented measurement equation is

$$
\left[\begin{array}{l}
\mathbf{G}_{\mathrm{a}} \\
\boldsymbol{\beta}_{\mathrm{m}}
\end{array}\right]=\left[\begin{array}{ccc}
\mathrm{C} & \mathrm{H}(\boldsymbol{\omega}) & 0_{4 \times 4} \\
0_{3 \times 3} & 0_{3 \times 16} & \mathrm{I}_{3 \times 3}
\end{array}\right]\left[\begin{array}{c}
\boldsymbol{\omega} \\
\mathbf{x} \\
\boldsymbol{\beta}
\end{array}\right]
$$

\section{COMPENSATION}

To complete the calibration process we need to perform its second stage; namely, compensation where we eliminate the estimated errors from the gyro readings. From Eq. (50.c) we obtain 


$$
\mathrm{C} \boldsymbol{\omega}_{\mathrm{r}}=\mathbf{G}_{\mathrm{a}}-\left[\begin{array}{lll}
\Omega^{\mathrm{m}} \mathrm{E} & \Omega^{\mathrm{k}} & \mathrm{I}_{4}
\end{array}\right]\left[\begin{array}{l}
\mathbf{d} \\
\mathbf{k} \\
\mathbf{b}
\end{array}\right]
$$

As mentioned before, $\mathbf{G}_{\mathrm{a}}$ is a vector of the gyro readings and $\boldsymbol{\omega}_{\mathrm{r}}$ is the correct angular velocity vector. However, we do not have $\omega_{\mathrm{r}}$ which is what we are trying to measure; therefore, to compute $\Omega^{\mathrm{m}}$ and $\Omega^{\mathrm{k}}$, which have to be computed using $\omega_{\mathrm{r}}$, we use the measured uncompensated angular rate vector. This vector is derived from the uncompensated gyro measurements which we called actual and denoted by $a$. Also, we do not have the actual values of $\mathbf{d}, \mathbf{k}$ or $\mathbf{b}$, but rather their estimate; therefore, using the values on hand Eq. (72.a) becomes

$$
\mathrm{C} \hat{\boldsymbol{\omega}}_{\mathrm{r}}=\mathbf{G}_{\mathrm{a}}-\left[\begin{array}{lll}
\Omega_{\mathrm{a}}^{\mathrm{m}} \mathrm{E} & \Omega_{\mathrm{a}}^{\mathrm{k}} & \mathrm{I}_{4}
\end{array}\right]\left[\begin{array}{l}
\hat{\mathbf{d}} \\
\hat{\mathbf{k}} \\
\hat{\mathbf{b}}
\end{array}\right]
$$

where $a$ denotes the actual values and $\wedge$ denotes estimated vectors. To obtain the compensated measurements of the angular rate vector define the A matrix, as follows:

$$
A=\left[\begin{array}{lll}
1 & 0 & 0 \\
0 & 1 & 0 \\
0 & 0 & \frac{1}{2}
\end{array}\right]
$$

It is easy to verify that

$$
\mathrm{AC}^{\mathrm{T}} \mathrm{C}=\mathrm{I}
$$

therefore pre multiplying Eq. (72.b) by $\mathrm{AC}^{\mathrm{T}}$ yields

$$
\hat{\boldsymbol{\omega}}_{\mathrm{r}}=\mathrm{AC}^{\mathrm{T}} \mathbf{G}_{\mathrm{a}}-\mathrm{AC}^{\mathrm{T}}\left[\begin{array}{lll}
\Omega_{\mathrm{a}}^{\mathrm{m}} \mathrm{E} & \Omega_{\mathrm{a}}^{\mathrm{k}} & \mathrm{I}_{4}
\end{array}\right]\left[\begin{array}{c}
\hat{\mathbf{d}} \\
\hat{\mathbf{k}} \\
\hat{\mathbf{b}}
\end{array}\right]
$$

\section{SIMULATION RESULTS}

In lieu of actual SC data, a simulator was developed to produce the gyro, reaction wheel, and star tracker data. Much care was devoted to the simulation since any dynamics errors would be perceived by the KF as a state error. First, the maneuver strategy was developed. An inertial period before any maneuver would facilitate the estimation of the gyro bias. The scale factors of each gyro could be estimated by a maneuver about that gyro axis. The misalignments could be estimated by the same scale factor maneuvers. An additional two maneuvers about the SC $\mathbf{X}$ and $\mathbf{Y}$ body axes, respectively, were added to assist in the alignment estimation of gyro 4 which senses rate about the body $\mathbf{Z}$ axis. Second, the maneuvers were modeled as a ramp-coast-ramp where the linear ramp time was 5 seconds. The rate profile was then obtained and $\dot{\boldsymbol{\omega}}$ was derived by simple subtraction. Third, the dynamics Eq. (59.a) was re-written in terms of $\dot{\mathbf{h}}$ and the ordinary differential equation (ODE) was solved using the $\boldsymbol{\omega}$ and $\dot{\boldsymbol{\omega}}$ generated above. Fourth, using the newly generated system momentum profile $\mathbf{h}$, the rate, $\boldsymbol{\omega}$, was determined from 

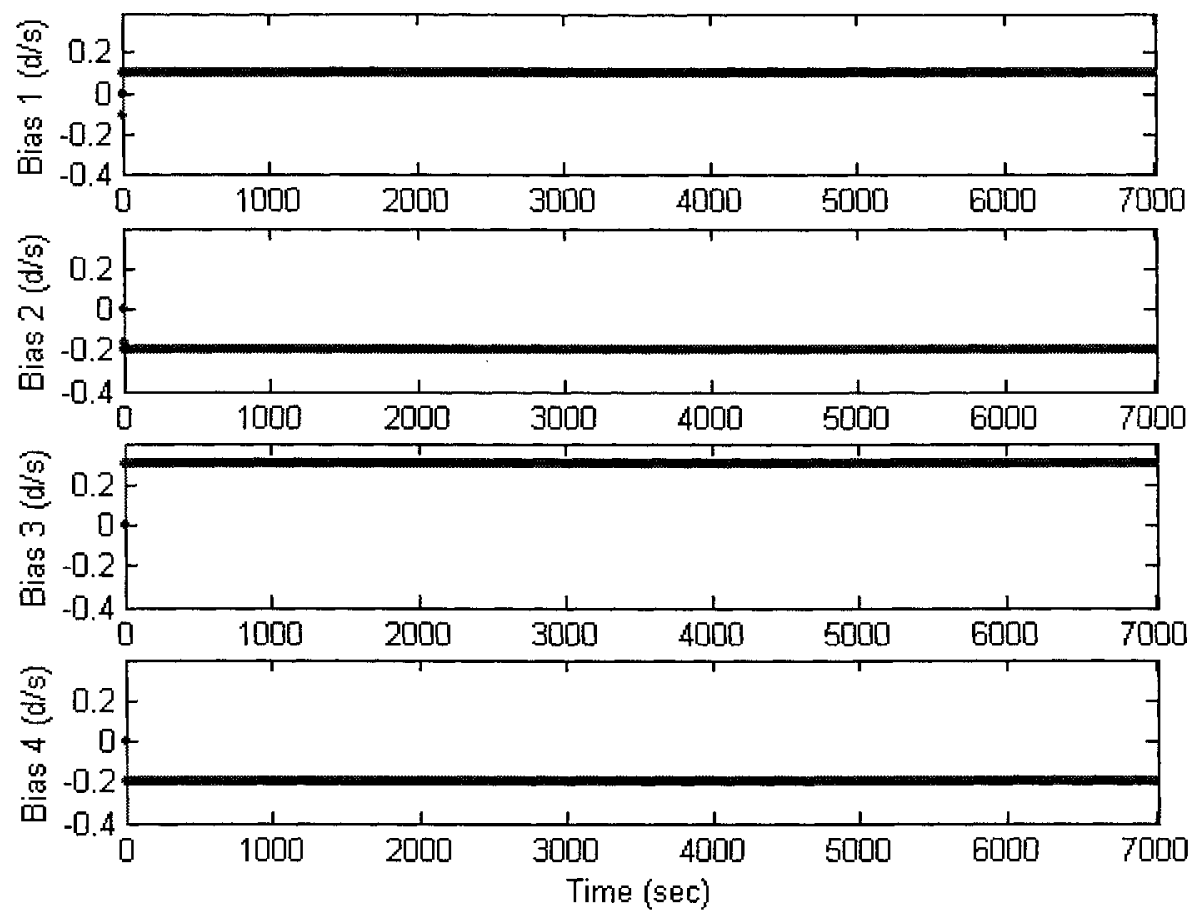

Fig. 5: Gyro Bias Estimate (bold) versus Truth (thin)
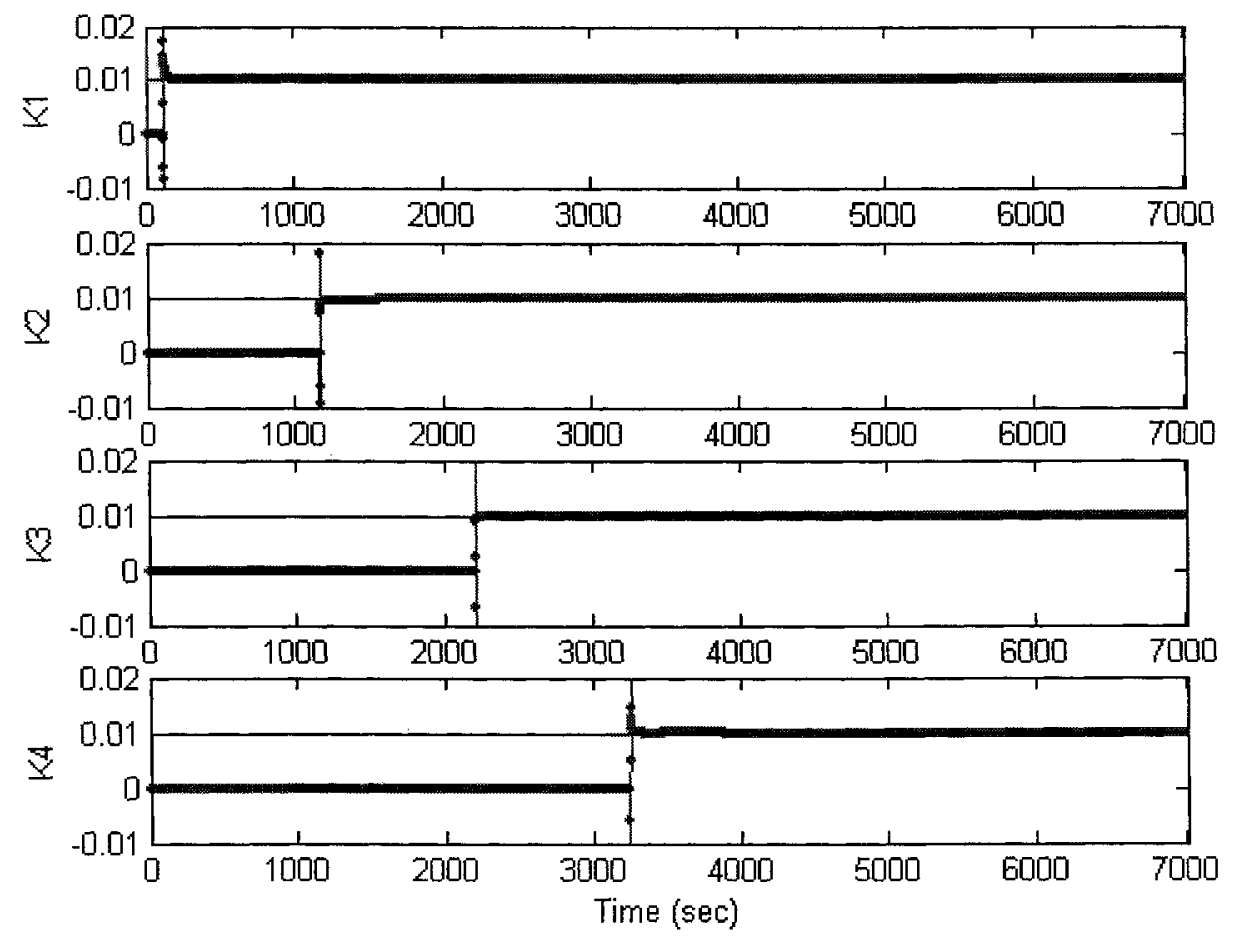

Fig. 6: Gyro Scale Factor Estimate (bold) versus Truth (thin) 

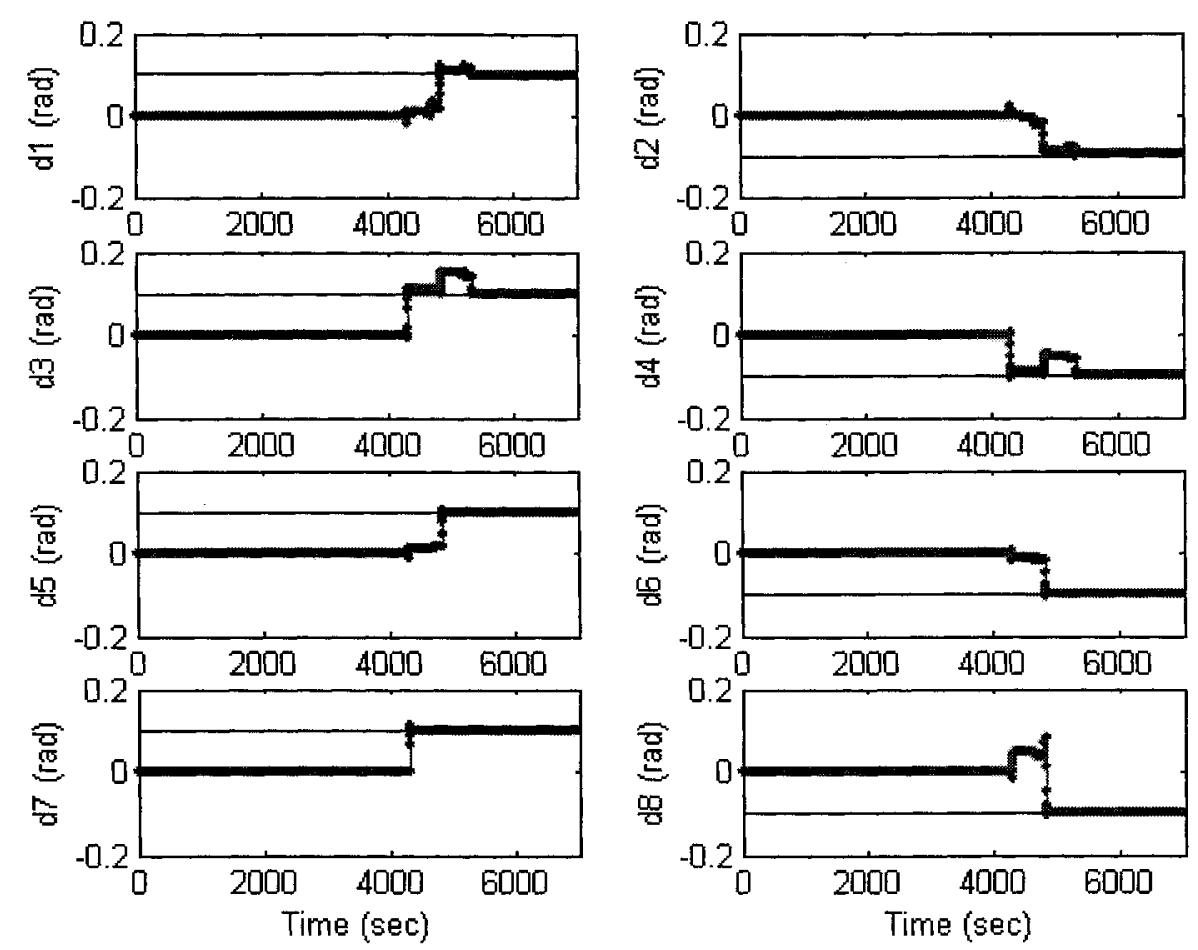

Fig. 7: Gyro Misalignment Estimate (bold) versus Truth (thin)

the ODE in Eq. (59.a). Fifth, using this $\boldsymbol{\omega}$, the quaternion was estimated using the $\dot{\mathbf{q}}$ portion of Eq. (61). Lastly, the body rate was resolved in the AQUA gyro frame by use of Eq. (50.c).

The KF was then executed on the simulated data. The biases were estimated using the initial inertial period. Also, to facilitate the bias estimation, the scale factor and misalignment parameter estimation was terminated by zeroing out their respective rows and columns in the state covariances and process noise. The scale factors for each gyro were then estimated using the respective maneuver about that axis and by zeroing out the influences of the other scale factors, the misalignments, and biases as described with the bias estimation. Lastly, the gyro misalignment slews, which were a repeat of the scale factor slews with the addition of $\mathbf{X}$ and $\mathbf{Y}$-axis maneuvers, were executed with the biases and scale factors zeroed out. All states were estimated with less than a $1 \%$ deviation from truth. The KF bias estimate can be seen in Fig. 5, followed by the scale factor estimate in Fig. 6, and lastly the misalignment estimate in Fig. 7.

\section{CONCLUSIONS}

In this paper we presented a new method of gyro calibration. Normally, we have to calibrate a cluster of three gyros whose sensitive axes are along the body axes. Here, the rate is read by four gyros only one of which is aligned along the body coordinate axes. Therefore, a new algorithm was devised for calibrating a quadruplet rather than the customary triad gyro set. In particular, a new model had to be developed for the gyro misalignment errors. Normally, the gyro outputs are used to supply data for a differential equation, which is solved in order to compute attitude. According to the new method the gyro outputs are also used as measurements, which are fed into a Kalman filter that estimates the gyro misalignment, scale factors, and biases. The new calibration algorithm was developed in particular for the 
calibration of the EOS-AQUA satellite gyros. The effectiveness of the new algorithm was demonstrated through simulations with error of each estimated parameter being less than $1 \%$.

\section{REFERENCES}

1. Chatfield, A.B., Fundamentals of High Accuracy Inertial Navigation, Vol. 174 Progress in Astronautics and Aeronautics, AIAA, 1997, pp. 93-106.

2. Bar-Itzhack, I.Y., and Harman, R.R., "True Covariance Simulation of the EUVE Update Filter," Proceedings of the 1989 Flight Mechanics Estimation Theory Symposium. NASA Conference Publication 3050, pp. 223-236.

3. Deutschmann, J.K., and Bar-Itzhack, I.Y., "Evaluation of Attitude and Orbit Estimation Using Actual Earth Magnetic Field Data," to be published in the Journal of Guidance, Control, and Dynamics.

4. NASA-Goddard Space Flight Center, Multimission Three-Axis Stabilized Spacecraft (MTASS), 553FDD-93/032R0UD0, 1933, pp. 3.3.2-1 - 3.3.2-10.

5. Gelb, A. (Ed.), Applied Optimal Estimation, MIT Press, Cambridge MA, 1974, p. 103.

6. Harman, R.R., and Bar-Itzhack, I.Y., "Pseudo-Linear and State Dependent Riccati Equation Filters for Angular Rate Estimation," Journal of Guidance, Control, and Dynamics, Vol. 22, No. 5, Sept.-Oct. 1999, pp. 723-725. (Engineering Note).

7. Bar-Itzhack, I.Y., "Classification of Algorithms for Velocity Estimation," Journal of Guidance, Control, and Dynamics, Vol. 24, No. 2, March-April 2001, pp. 214-218.

8. Azor, R., Bar-Itzhack, I.Y., Deutschmann, J.R. and Harman, R.R., "Angular-Rate Estimation Using Delayed Quaternion Measurements," AIAA Guidance, Navigation, and Control conference, Portland, OR, August 9-11, 1999. 